\title{
The Rejuvenation of the Aged Central Lower Face: A Contemporary Perspective
}

\author{
Fred G. Fedok, MD, FACS ${ }^{1}$ \\ ${ }^{1}$ Department of Surgery, University of South Alabama, Mobile, Alabama \\ Facial Plast Surg 2019;35:121-128.
}

\author{
Address for correspondence Fred G. Fedok, MD, FACS, Fedok Plastic \\ Surgery, 113 East Fern Avenue, Foley, AL 36535 \\ (e-mail: drfredfedok@me.com).
}

\begin{abstract}
Keywords

- central lower face

- biologicals

- platelet-rich plasma

- fat transfer

- marionette lines

Aging of the central face is typified by several common characteristics such as generalized volume loss, the appearance of a mental crease and marionette lines, thinning of the lips, and fixed and dynamic rhytids. These result from several processes such as atrophy of adipose and bone, changes in the support of the mandibular septum, and a loss of skin elasticity. There are many methods available for the improvement and rejuvenation of this facial anatomical area. Options include traditional surgical techniques, the application of volume and the use of laser, light, and energy-based devices. The recent application of biological interventions offers the possibility of cellular-based therapies to usher in a new era of true tissue regeneration. Optimal correction of the abnormalities may be accomplished through an understanding and identification of the causes of the observed abnormalities and targeting with the best available intervention. There are a growing number of techniques and technologies to accomplish this.
\end{abstract}

As the average age of the population continues to increase, ${ }^{1,2}$ there has been a growing demand for facial rejuvenation procedures. In response to this demand for facial rejuvenation, there has been an introduction of a widening array of techniques, methods, and technologies to accomplish this.

The past 50 years have seen a tremendous improvement in our understanding of the processes integral to facial aging. In the past, surgical attention appeared to be largely on the loss of elasticity and sagging of facial skin, whereas now there is a greater appreciation of the role of involution-based changes in facial soft tissue suspensory elements, soft tissue volume, and skeletal mass. ${ }^{3}$ In turn, there has been a development of a spectrum of methods to reverse these age-related changes with the replenishment of volume, repositioning of facial soft tissue elements, and biological interventions.

Much has been written about the sagging of the eyebrows, the descent of midface structures, and the sagging of the neck tissues that come with aging. Relatively less has been written about the aging of the central lower face. This has been due, in part, to the previous paucity of methods to effectively rejuvenate this anatomical area where aging is marked by deflation and fixed rhytids around the perioral area.

This important anatomical region includes the lips, the jawline, the chin, the subnasal, and the mandible. Although arguably not as important as the upper face in social interactions, the lips and associated regional structures hold a similarly crucial role in facial discernment and social interface. An examination of the central lower facial areas of aging patients will suggest that this area, due to a relative disproportionate greater impact of volume loss compared with other facial regions, takes on an aged appearance in a more accelerated fashion than some of the other parts of the face.

Many of the past approaches to the central lower face have been unsatisfactory, that is, the recommendation to deeply dermabrade the perioral rhytids, resulting in unsightly local hypopigmentation. The contemporary recognition of the need for volume and contemporary methods of skin tightening has opened up a newer chapter in the rejuvenation of the central lower face.

\section{The Aesthetics of the Central Lower Face}

The central lower facial region includes the upper and lower lips, as well as the chin. The central lower facial region encompasses the inferior one-third of the horizontal facial proportions and can be further subdivided, with the upper lip accounting for one-third and the lower lip and chin accounting for two-thirds of the lower facial division. ${ }^{4,5}$
Issue Theme Aesthetic Treatment of the Jawline and Perioral Area; Guest Editor: S. Randolph Waldman, MD
Copyright (C) 2019 by Thieme Medical Publishers, Inc., 333 Seventh Avenue, New York, NY 10001, USA. Tel: +1(212) 584-4662.
DOI https://doi.org/ 10.1055/s-0039-1683857. ISSN 0736-6825. 
Subtle transitions in contour, texture, and color produce the form of the lips, the chin, and the transition of these central facial regions to the lower aspect of the lateral cheek. The lower extent of these contours merges with the inferior mandibular border. 6

\section{Pertinent Anatomy}

The involutional changes associated with aging in the lower face have only been recently delineated ${ }^{7}$ and involve every aspect of the local anatomy including the skin, the subcutaneous tissues, and the bone. The anatomical mechanisms underlying the age-related changes of the mandible contour include atrophy of the superior and inferior mandibular fat compartments, dehiscence of the mandibular septum causing the superior and inferior fat compartments to move downward to the neck, bone resorption, and skin laxity.

The support of the lower facial soft tissues is borne principally by the mandible. The inferior mandibular border serves as the platform for the insertion of the lower facial musculature, ligaments, and mandibular septum. These attachments create the structural soft tissue scaffolding for the overlying subcutaneous tissues, producing the contour of the lower face and mandibular border. This anatomical complex moves in unison with the motion of the mandible with speaking, facial expressions, and eating. In youth, there is a fullness and continuity of these structures, resulting in the idealized contours. However, the involutional changes that occur with aging result in the disturbance of the smooth lower facial contours and cause the appearance of lumps, bulges, and depressions.

Atrophy of the mandibular bone occurs in a manner similar to what happens to other projecting regions of the facial skeleton, such as the chin and the orbital rims. ${ }^{8}$ This mandible atrophy is irregularly distributed along various locations of the mandibular margin, depending on the underlying biomechanical forces impacting the mandible in that particular region. These forces include those generated by muscular movement, gustation, and speaking, and those imposed by the overlying skin and soft tissues. The atrophic mandibular remodeling is especially pronounced in the area of the anterior mandible immediately inferior to the mental foramen, resulting in an anatomical boney depression known as the anterior mandibular groove. This depression of the bone is situated just anterior to the attachments of the mandibular ligament. ${ }^{6}$

Soft tissue changes in this region also occur alongside the slow resorption of the bone. There is partial dehiscence of the muscular insertions and fascial structures in the area creating a worsening misdirection of the local muscular actions. This results in a compensatory resultant hypertrophy of other portions of the muscular components that are still firmly attached. The pertinent muscles in the area include the depressor anguli oris (DAO), the mentalis muscle, and the depressor labii inferioris muscle. ${ }^{7}$ Behind the DAO muscle, inserting into the skin, is the mandibular ligament.

With the atrophy of the overlying fat compartments and the hypertrophy of these muscles, one notes changes in the lower central face, which include the deepening of the melomental folds, the mental crease, and the labiomandibular creases.
The delineation of the fat compartments of the lower face is a relatively recent concept and includes the identification of the superior and inferior mandibular fat compartments that extend over the inferior mandibular border. The lower face's fat compartments have two layers: the superficial fat compartment and the deep fat compartment. ${ }^{3}$ The mandibular septum is a membranous septum that separates the two compartments located over the mandibular edge from the submandibular fat compartment. ${ }^{6}$ Fibers from the platysma intermingle with the mandibular septum and are inserted at the anterior border of the mandible.

The conceptualization of these fat compartments has allowed a better understanding of the age-associated changes in the lower face. The age-related deflation of these fat compartments and fat malposition cause a distortion in the soft tissue laden mandibular border that typifies a healthy youthful appearance. The resultant distortions result in anatomical characteristics that we identify as jowling, the appearance of marionette lines, and a deepening prejowl sulcus.

Resorption of subcutaneous fat and colloidal fullness, as well as bony resorption, causes the lower facial musculature to no longer be contiguous with the lateral and submental jawline. The chin becomes defined by the volume loss in the prejowl sulcus, labiomental fold, labiomental sulcus, and submental crease. People also commonly become aware of a progressive worsening of the skin contour with dimpling and shadowing. In its more extreme form, this effect can be described as a peau d'orange. The "witch's chin" results from a further severe loss of volume and support.

\section{Age-Related Changes to the Local Anatomy: What May Be Anatomically Different from the Other Parts of the Face?}

For many patients, the involutional changes centered about the lower central face appear earlier than those in other parts of the face, such as the cheeks and forehead (-Fig. 1). This may be secondary to a disproportionate greater loss of

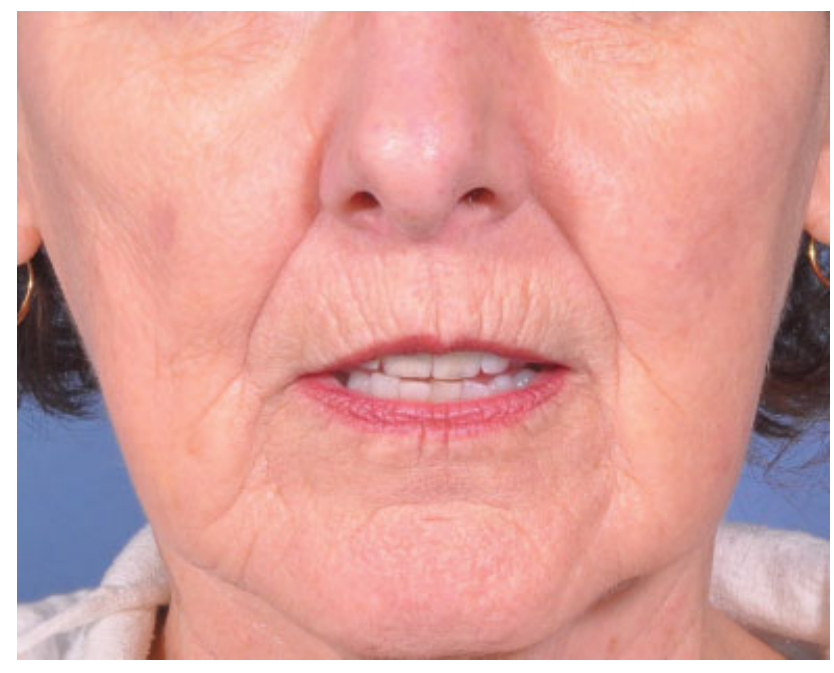

Fig. 1 Clinical image of patient depicting many of the characteristics of the aging central lower face: fat depletion of the anatomical region, prominent perioral fixed rhytids, thinning of the lips, prominence of the prejowl sulcus, and marionette lines. 

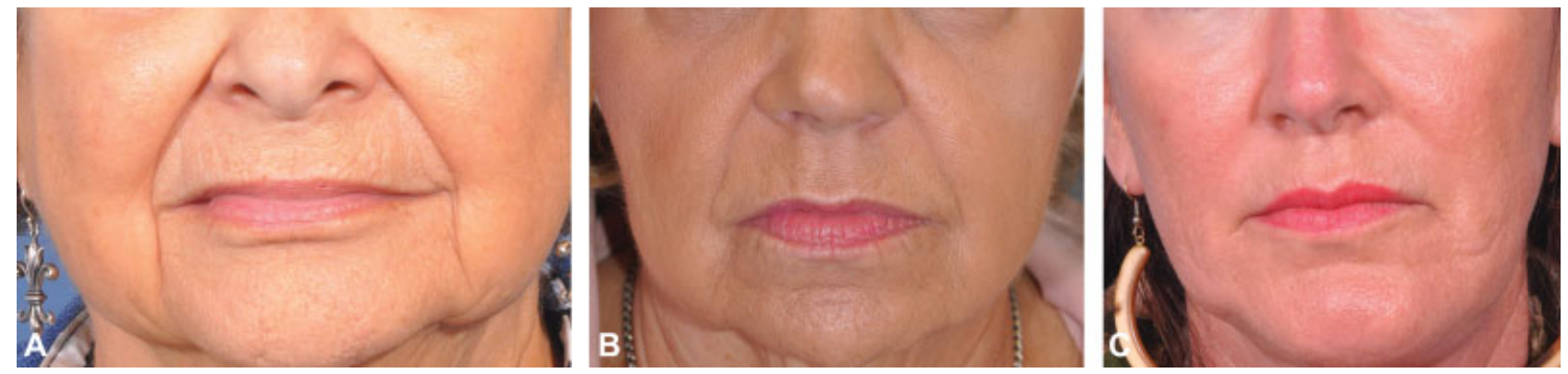

Fig. 2 (A-C) Clinical images of the central lower face of three patients at three different ages demonstrating varied manifestations of the characteristics noted in - Fig. 1.

volume and support that occurs in this region secondary to changes in adipose and bone. It may be further compounded by the relatively fixed nature of the muscles and soft tissues around the lips and of the overlying skin. The muscles here do not move bones around joints. They instead move the overlying skin and other soft tissue elements in reference to their underlying attachments to the bone. Because of the confluence of muscle attachments to the orbicularis muscle and to the soft tissue of the lips and the chin, the loss of fatty volume in the area is more prone to cause depressions, wrinkling, and fixed rhytids.

Patient images demonstrating different permutations of the aforementioned deleterious characteristics of aging of the central lower face are depicted in $\mathbf{- F i g} . \mathbf{2}$.

\section{Why One Should Consider a Multidimensional Approach in the Rejuvenation of the Central Lower Face}

As in most areas of the face, a multidimensional approach to rejuvenation should be considered given the multidimensional nature of aging in the region. Aging results in changes to the underlying bone, the muscles, the adipose, and the skin. Some of these changes are static, such as the static state of the deflated lips, and some of them are dynamic, such as the dimpling of the chin that occurs with movement. The involution of form and function occurs along different timelines and is because of intrinsic and extrinsic factors. The term multidimensional is used to emphasize the complexity of aging of the facial tissues, which is because of a multitude of past and ongoing processes. Aging involves changes in every tissue layer and in the facial area of interest. Some of the deleterious changes are functional and some are anatomical. It is strongly suggested that to "rejuvenate" a facial area, several interventions are needed to bring optimal changes. By adopting a multimodality approach, one can best remedy the various components of the local aging characteristics. For instance, in an older patient, it is essentially fruitless to attempt to rejuvenate the prejowl sulcus without the application of volume. The underlying contour deficiency is secondary to the loss of both bone and soft tissue. The deficiency is further accentuated because of the juxtaposition of the jowl adjacent to the area of volume loss. Hence, an optimal correction usually involves not only tissue repositioning, that is, through face-lifting but also the application of volume.

\section{A General Approach to the Lower Facial Third}

A fundamental list of techniques might be suggested to be considered necessary for the optimal rejuvenation of a frequently encountered constellation of age-related characteristics in the central lower facial region of the aging patient. These characteristics include the presence of prejowl and perioral volume loss, and fixed and dynamic rhytids at the commissures and surrounding the lips. On examination, one will see peau d'orange changes around the lips and chin, deflated and thin lips, and an exacerbation of marionette lines (-Fig. $\mathbf{3}$ ).

To improve these aged characteristics, several changes have to be made in the local tissues: volume must be adequately applied, and the correction of fixed rhytids in the regional skin will have to be induced through dermal and epidermal remodeling. Volume will have to be added in the prejowl sulcus, as well as the chin and the white and red lips. Volume can be autogenous tissue, alloplastic hard implants, and injectable materials, depending on the exact location. Correction of the fixed rhytids will require some method to efface the etched and fixed rhytids, such as the use of
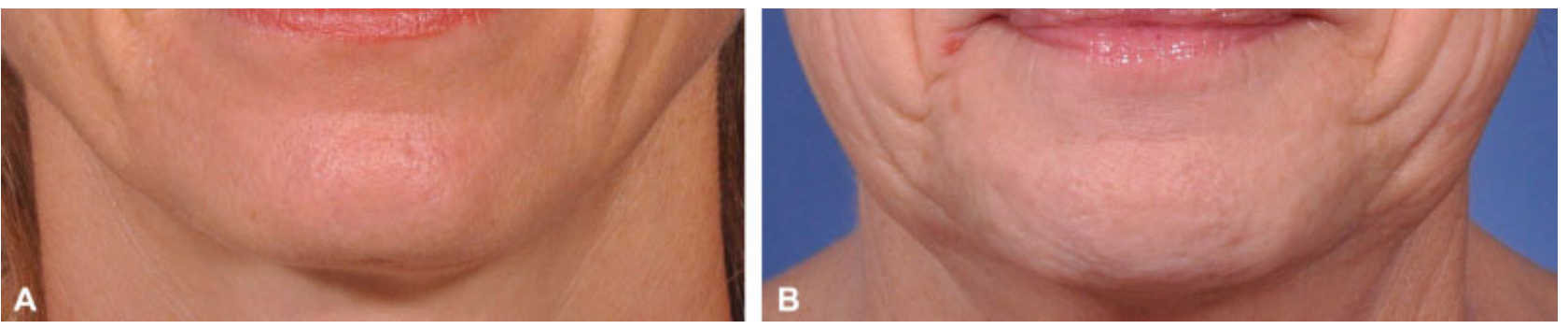

Fig. $3(A, B)$ Clinical images of the central lower face of two patients demonstrating peau d'orange changes secondary to volume loss at the chin and adjacent tissues. 
alloplastic or autogenous injectables or some method to improve skin, via dermal collagen remodeling including lasers, microneedling, and other energy-based devices.

\section{Specific Modalities}

\section{Biologicals}

There are many autogenous biological materials that are currently being used for facial regeneration and rejuvenation. Fat transfer, nanofat injection, and the use of plateletrich plasma have been widely published for their use for cosmetic purposes. ${ }^{9,10}$

Autogenous fat has been used in various forms and preparations. Processing of the autogenous fat is performed through methods as simple as routine liposuction and at times through a more complicated process by passing harvested fat through smaller cannulas or passing it through several filters to produce a finer material for injection.

Standard "macrofat" is used primarily for volume restoration. For many practitioners, it is considered superior or at least equivalent to other injectable materials for restoring volume loss. Fat transfer is performed both as an isolated procedure and with other procedures such as rhytidectomy. Reports are variable regarding the retention of the fat. By adherence to several principles of reliable fat harvesting and processing, it is reported that up to 50 to $75 \%$ of injected fat is retained. ${ }^{11-14}$

In this facial region, fat can be used to restore volume to the lips themselves, both the red lip and the white lip. It can be used to fill the volume loss at the prejowl sulcus. The injected volume will help minimize marionette lines. It can be injected in the chin to minimize some of the deleterious effects of fat wasting in the area.

The benefits of the injection of fat appear to extend beyond the simple placement of volume. The accompanying adipose-derived stem cells are believed to favorably influence the local tissues through the action of various growth factors and other bioactive substances, causing an improved skin texture and elasticity. ${ }^{15,16}$

Nanofat, on the other hand, is derived from the morsilization of a fat aspirate. Nanofat has been described as being devoid of viable fat cells and instead having a high concentration of fat-derived stem cells. Stem cells isolated from lipoaspirates have demonstrated in vitro differentiation into adipogenic, osteogenic, chondrogenic, myogenic, cardiomyogenic, and neurogenic lineages. There is evidence that adiposederived stem cells secrete vascular endothelial growth factor, hepatocyte growth factor, and transforming growth factor- $\mathrm{b}$ in the presence of stimuli such as hypoxia and strongly influence cellular differentiation, promote angiogenesis and wound healing, and potentially aid in new tissue growth and development. Nanofat is therefore not injected for its volume attributes but instead for the stem cell attributes. It is believed that it may take several months until one is able to note differences in the local tissues ${ }^{15-21}$ (- Figs. 4, 5).

\section{Platelet-Rich Plasma}

Platelet-rich plasma is frequently used in the repair and rejuvenation of soft tissues. The literature is replete with reports of the benefits in the treatment of rhytids, scars, alopecia and other medical issues. There is good evidence that the addition of platelet-rich plasma will improve the retention of transferred fat. Platelet-rich plasma is derived from blood drawn from the patient. The blood is processed through centrifugation with various proprietary gels to produce a serum fraction with a high concentration of platelets. This platelet-rich plasma fraction has been found to contain a large number of beneficial growth factors and cytokines. When injected into tissues there have been shown to have several benefits in mechanisms of healing and tissue regeneration. Similar to nanofat, the injection of platelet-rich plasma is not performed for volume restoration but instead for the injection of growth factors and other beneficial biologic messengers ${ }^{10,22,23}$ (- Fig. 6).
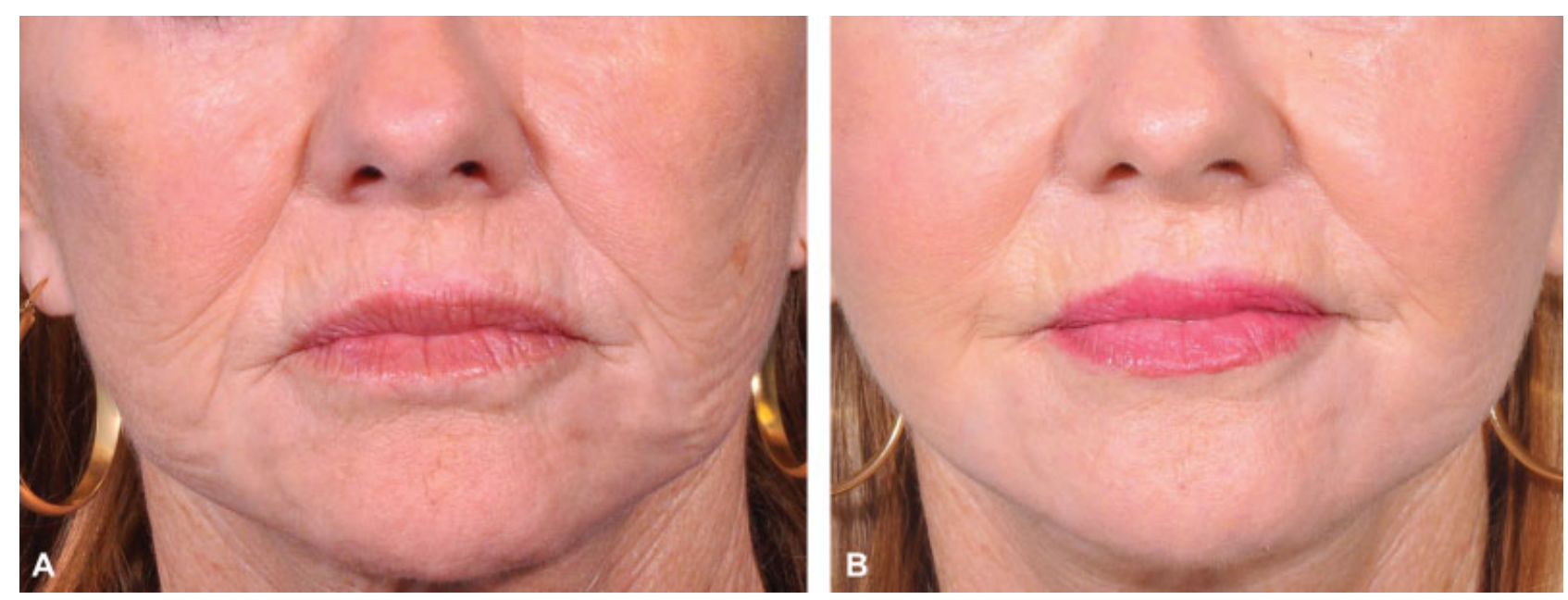

Fig. 4 (A,B) Clinical images of the central lower face of the patient who underwent lower facelift, nanofat injection, fat transfer, and fractional $\mathrm{CO}_{2}$ resurfacing. The goal was to initiate an improvement in the prejowl sulcus, the perioral rhytids, and the deflated local appearance. Note the combined benefits of soft tissue repositioning from the facelift, improvement of etched fine lines with nanofat, volume restoration from the macrofat transfer, and improvement in the skin texture with the laser resurfacing. (A) Preoperative and (B) postoperative 6 months images. 

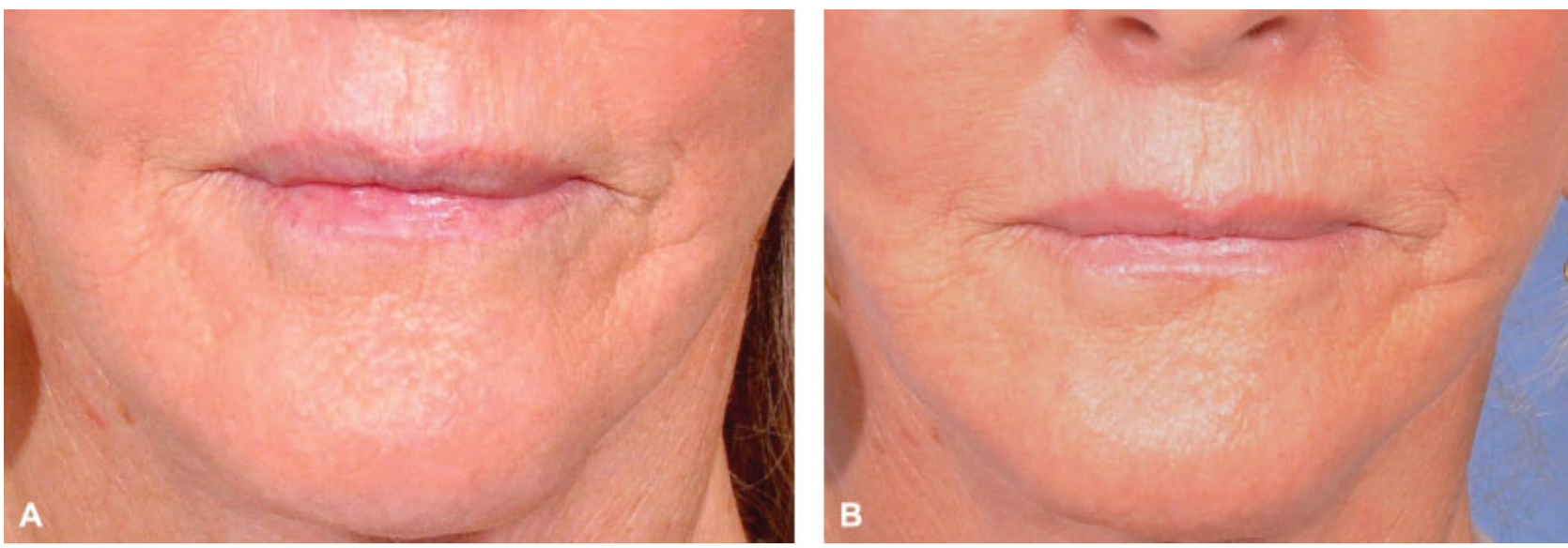

Fig. 5 (A,B) Early results of the injection of nanofat alone at 4 months. Note improvement in fine rhytids. (A) Preoperative and (B) postoperative images.

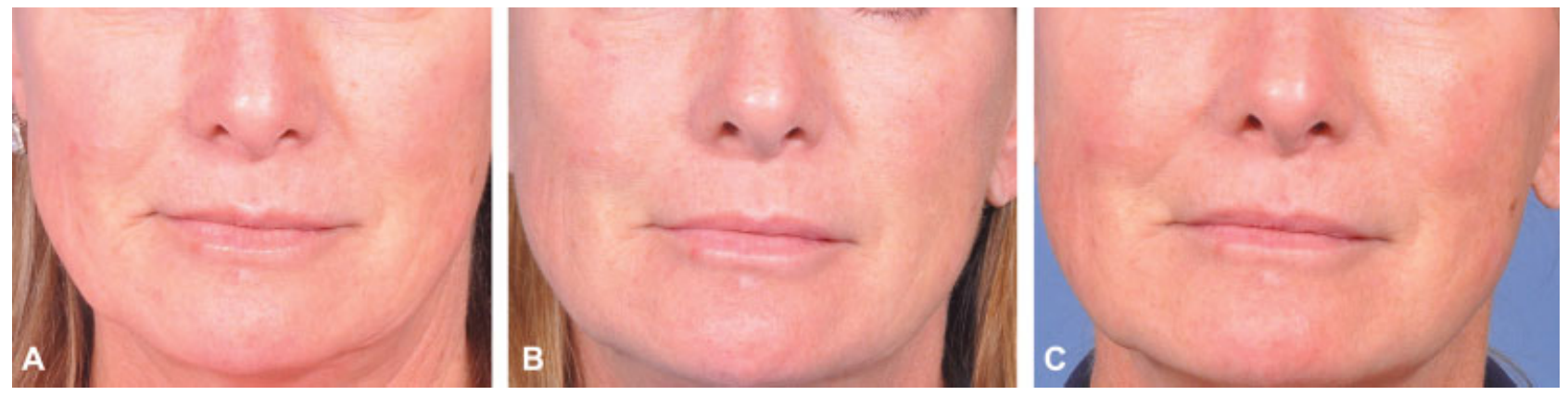

Fig. 6 (A-C) The results of radiofrequency microneedling, concurrent skin surface application, and injection of platelet-rich plasma into fine-etched perioral rhytids in two sessions. (A) Preoperative, (B) 3 months postoperative, before the second session, and (C) 7 months postoperative from the first session images.

\section{Skin Tightening: Laser, Light, and Energy-Based Devices}

Skin tightening in the central lower face may be accomplished with a large number of energy-based technologies. Many of these can be categorized into epidermal sparing and ablative.

Skin-tightening technologies that are generally ablative include full ablative and fractional ablative laser technologies, plasma devices, chemical peels, and dermabrasion.

Skin-tightening technologies that are generally epidermal sparing include nonablative lasers, subcutaneous plasma devices, and various radiofrequency and ultrasonic frequency devices. A category of technologies that are general epidermal sparing but do penetrate the epidermis include microneedle and radiofrequency microneedle technologies. One important caveat is that the effect on the epidermis following a radiofrequency microneedling session typically last less than 24 hours and does not cause epidermal ablation. The principle that is being forwarded here is one should treat what must be treated and preserve what does not need to be treated.

There are various opinions regarding the best technologies to be used. Each modality carries with it a list of positive and negative attributes. In general, if the surface of the skin demonstrates considerable photodamage and is in need of being refreshed, then the use of ablative technologies is logical and will produce changes in the surface the skin and create dermal tightening for improvement of fixed rhytids. If, on the other hand, the surface of the patient's skin does not show extensive photodamage, then one might want to create favorable dermal tightening through the use of epidermal-sparing technologies such as one of the radiofrequency technologies or microneedling. Do not ablate the epidermis if it is not necessary. There are other technologies to create tightening. The epidermal-sparing and ablative technologies can also be used in combination. ${ }^{22,24-27}$

\section{Soft Tissue Repositioning}

A central premise of this manuscript is that this anatomical facial area should be approached with a multidimensional orientation for rejuvenation. Multiple modalities, techniques, and processes should be considered to be useful, depending on which components of aging are manifested in the particular patient. The surgeon should have an extensive palette of tools available, such as injectables, neuromodulators, biologicals, energy-based technologies, and, of course, surgery.

Correction of the prejowl sulcus is accomplished by the repletion of volume at the sulcus and also by correction of the jowl itself. The combined areas of the midface and the lateral lower face occupy the areas lateral to the melolabial fold and the central lower face. The lower portion of the melolabial fold forms the jowl at mandibular border. It frames the central lower face.

To optimally correct the jowl and the prejowl sulcus, a facelift technique has to be used that will reposition the midface and lift the jowl containing portion of the superficial musculoaponeurotic system (SMAS). This will improve both the lower face and the neck ${ }^{28-30}$ (-Fig. 7). 

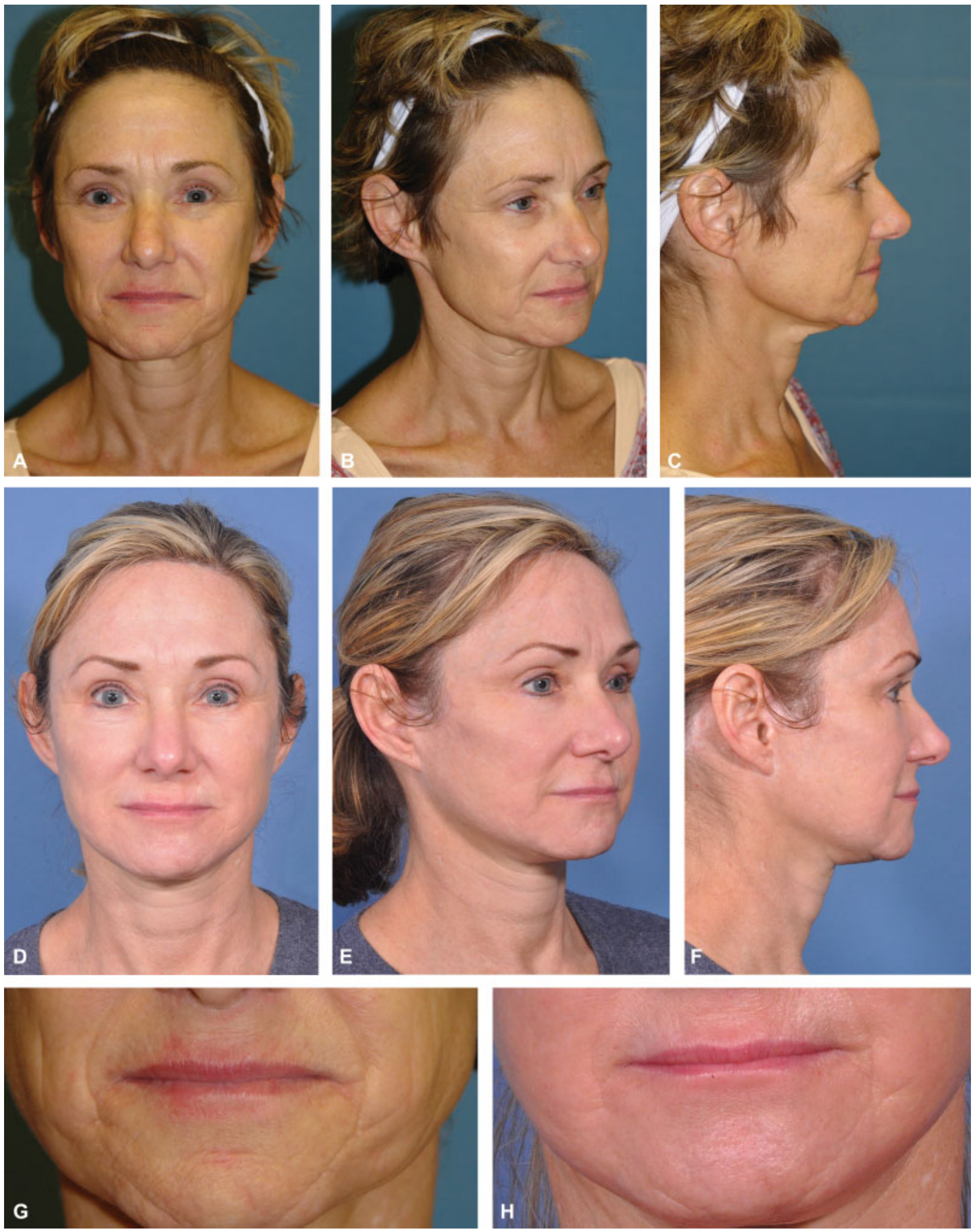

Fig. 7 (A-H) Images of patient who has undergone several procedures pertaining to her lower face, including lower facelift with platysmaplasty, $35 \%$ trichloroacetic acid chemical peel/Jessner's peel, fat transfer (2014), transpalpebral midface lift, fat transfer, fractional erbium resurfacing, and radiofrequency microneedling (2017). (A-C) Preoperative, (D-F) postoperative at 7 months after final procedures, (G, H) close-up images of the lower central face at same timeframes. 

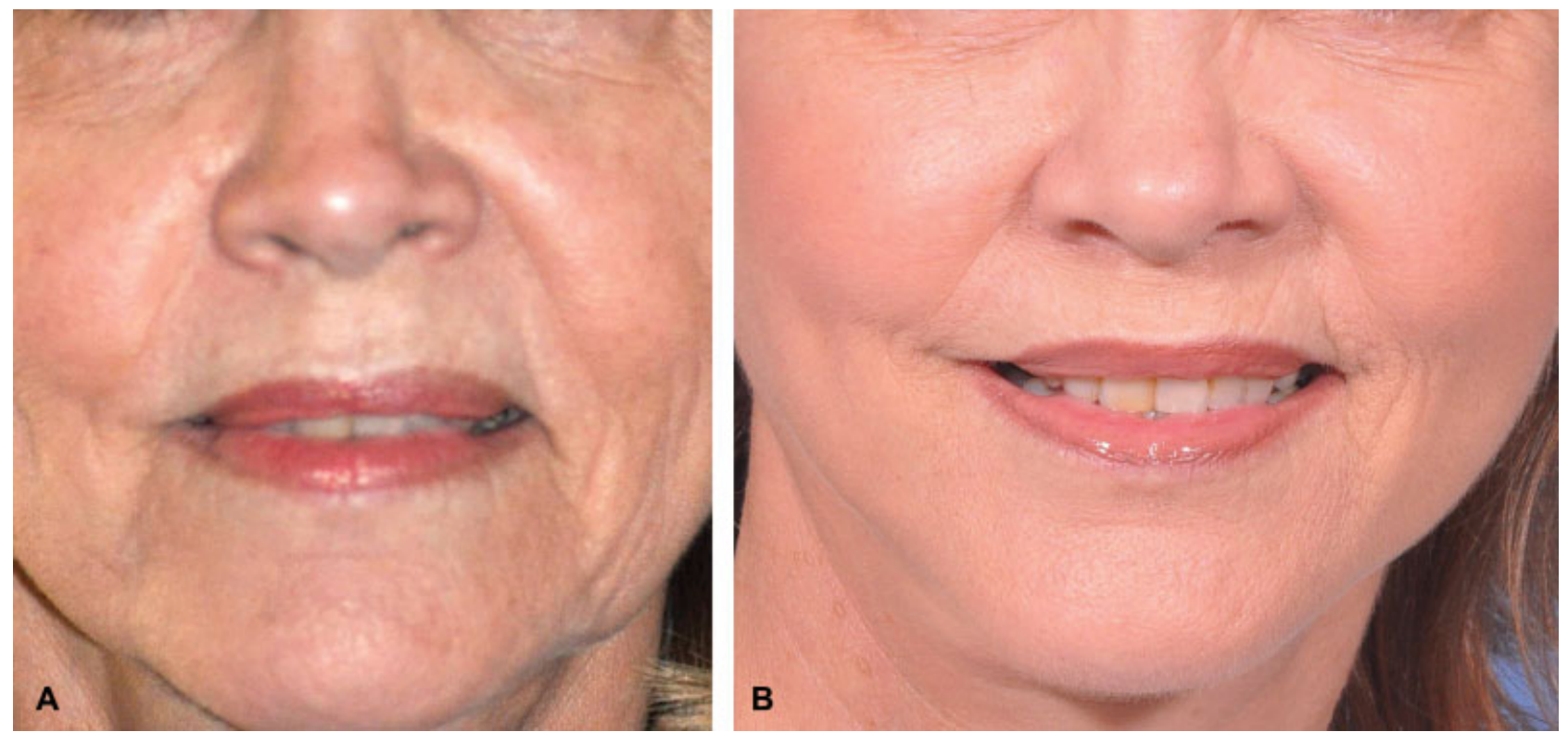

Fig. 8 (A,B) Patient images 11 months after the performance of the "bullhorn" lip lift with concomitant deep plane facelift.

\section{Neuromodulators}

Effective rejuvenation frequently requires the use of many modalities. In spite of the key roles of the replacement of volume and use of other technologies in the rejuvenation of the central lower face, the need for neuromodulators as a therapeutic and preventative tool continues. Neuromodulators can be used for the improvement of dynamic and fixed perioral rhytids, turned-down oral commissures, marionette lines, and dimples and depressions over the chin.

\section{Solid Implants}

Depending on the patient's anatomy, solid implants continue to play an important role in the correction of the prejowl sulcus and other mandibular anatomical abnormalities. ${ }^{11}$

\section{Focal Lip Procedures}

Several surgical procedures have been described for the enhancement of the lips. Advancements of the lip vermillion can be performed on the upper and lower lips to increase the relative amount of vermillion show. ${ }^{31}$ The lip lift procedure increases the upper lip's red lip show and also decreases the length of the deflated curtainlike upper $\operatorname{lip}^{32}$ ( - Fig. 8).

\section{Conclusion}

Aging of the central lower face is part of the mosaic of general facing aging. It exhibits all of the stigmata of aging in other areas of the face. Its underlining involutional processes are also similar. It does demonstrate some unique characteristics based largely on the unique facets of its local anatomy compared with other aspects of the face. It has many areas where the muscles insert closely to the overlying skin and hence volume loss has a greater impact on the skin appearance, that is, around the lips.

Traditional management of aging of the central lower face has evolved because of the innovations in surgical techniques and technologies. Prior to 30 years, the rejuvenation of this area was limited only to changes that could be produced with surgical procedures, hard implants, and resurfacing procedures that were largely limited to chemical peels and dermabrasion.

In the interim, the use of injectable fillers, neuromodulators, and many energy-based devices has allowed interventions to be directed at different facets of the local aging. Muscle actions could be altered and could diminish their impact on the overlying skin, and deficiencies in local volume could be addressed. Lasers and other energy-based devices could actually influence dermal remodeling and tighten the skin.

More recently, the use of fat transfer has been shown to have many benefits applicable to facial rejuvenation. A key ingredient of facial rejuvenation, the restoration of youthful volume, has been remarkedly forwarded through the use of fat transfer. Other biologicals such as platelet-rich plasma, nanofat, and stem cells have shown some promise in domains of regeneration and rejuvenation. These biological interventions will have to be approached prudently, at this time, since large well-designed investigations into their limitations and benefits are still to be convincingly performed. They show considerable promise and appear to be a doorway to amazing future innovations and benefits. Perhaps, the terms "cell-based" or "cellular therapies" will be most fitting since the goal is to rejuvenate through the application of "cells" and their associated bioactive substances.

In the meantime, keep a watchful eye on the developments that are being reported real time in these discussed areas. The author is among those who believe that we are on the threshold of some of the most meaningful innovations in plastic surgery and regenerative medicine.

Funding

The author has no relevant financial disclosures regarding this manuscript. 
Conflict of Interest

None declared.

\section{References}

1 Cacioppo JT, Cacioppo S. The population-based longitudinal Chicago Health, Aging, and Social Relations Study (CHASRS): study description and predictors of attrition in older adults. Arch Sci Psychol 2018;6(01):21-31

2 Vogelsang EM, Raymo JM, Liang J, Kobayashi E, Fukaya T. Population aging and health trajectories at older ages. J Gerontol B Psychol Sci Soc Sci 2017 (e-pub ahead of print). doi:10.1093/ geronb/gbx071

3 Rohrich RJ, Pessa JE. The anatomy and clinical implications of perioral submuscular fat. Plast Reconstr Surg 2009;124(01): 266-271

4 Perkins SW, Sandel HD IV. Anatomic considerations, analysis, and the aging process of the perioral region. Facial Plast Surg Clin North Am 2007;15(04):403-407, v v .

5 Ridley MV. SM. Aesthetic facial proportions. In: Papel ID, ed. Facial Plastic and Reconstructive Surgery. 3rd ed. New York, NY: Thieme; 2009:119-113

6 Reece EM, Pessa JE, Rohrich RJ. The mandibular septum: anatomical observations of the jowls in aging-implications for facial rejuvenation. Plast Reconstr Surg 2008;121(04):1414-1420

7 Braz A, Humphrey S, Weinkle S, et al. Lower face: clinical anatomy and regional approaches with injectable fillers. Plast Reconstr Surg 2015;136(5, Suppl):235S-257S

8 Romo T, Yalamanchili H, Sclafani AP. Chin and prejowl augmentation in the management of the aging jawline. Facial Plast Surg 2005;21(01):38-46

9 Taub AF, Pham K. Stem cells in dermatology and anti-aging care of the skin. Facial Plast Surg Clin North Am 2018;26(04):425-437

10 Lin J, Sclafani AP. Platelet-rich plasma for skin rejuvenation and tissue fill. Facial Plast Surg Clin North Am 2018;26(04):439-446

11 Fedok FG, Mittelman H. Augmenting the prejowl: deciding between fat, fillers, and implants. Facial Plast Surg 2016;32 (05):513-519

12 Ransom ER, Antunes MB, Bloom JD, GrecoT. Concurrent structural fat grafting and carbon dioxide laser resurfacing for perioral and lower face rejuvenation. J Cosmet Laser Ther 2011;13(01):6-12

13 Sykes JM, Tapias V, Pu LL. Autologous fat grafting viability: lower third of the face. Facial Plast Surg 2010;26(05):376-384

14 Glasgold M, Lam SM, Glasgold R. Autologous fat grafting for cosmetic enhancement of the perioral region. Facial Plast Surg Clin North Am 2007;15(04):461-470, vi

15 Xu P, Yu Q, Huang H, Zhang WJ, Li W. Nanofat increases dermis thickness and neovascularization in photoaged nude mouse skin. Aesthetic Plast Surg 2018;42(02):343-351

16 Uyulmaz S, Sanchez Macedo N, Rezaeian F, Giovanoli P, Lindenblatt N. Nanofat grafting for scar treatment and skin quality improvement. Aesthet Surg J 2018;38(04):421-428
17 Jan SN, Bashir MM, Khan FA, et al. Unfiltered nanofat injections rejuvenate postburn scars of face. Ann Plast Surg 2019;82(01): 28-33

$18 \mathrm{GuZ}$, Li Y, Li H. Use of condensed nanofat combined with fat grafts to treat atrophic scars. JAMA Facial Plast Surg 2018;20(02):128-135

19 Bi HS, Zhang C, Nie FF, Pan BL, Xiao E. Basic and clinical evidence of an alternative method to produce vivo nanofat. Chin Med J (Engl) 2018;131(05):588-593

20 Lo Furno D, Tamburino S, Mannino G, et al. Nanofat 2.0: experimental evidence for a fat grafting rich in mesenchymal stem cells. Physiol Res 2017;66(04):663-671

21 Tonnard P, Verpaele A, Peeters G, Hamdi M, Cornelissen M, Declercq H. Nanofat grafting: basic research and clinical applications. Plast Reconstr Surg 2013;132(04):1017-1026

22 Ibrahim MK, Ibrahim SM, Salem AM. Skin microneedling plus platelet-rich plasma versus skin microneedling alone in the treatment of atrophic post acne scars: a split face comparative study. J Dermatolog Treat 2018;29(03):281-286

23 Alexander RW. Biocellular regenerative medicine: use of adiposederived stem/stromal cells and it's native bioactive matrix. Phys Med Rehabil Clin N Am 2016;27(04):871-891

24 Asif M, Kanodia S, Singh K. Combined autologous platelet-rich plasma with microneedling verses microneedling with distilled water in the treatment of atrophic acne scars: a concurrent splitface study. J Cosmet Dermatol 2016;15(04):434-443

25 El-Domyati M, Abdel-Wahab H, Hossam A. Microneedling combined with platelet-rich plasma or trichloroacetic acid peeling for management of acne scarring: a split-face clinical and histologic comparison. J Cosmet Dermatol 2018;17(01):73-83

26 Faghihi G, Keyvan S, Asilian A, Nouraei S, Behfar S, Nilforoushzadeh MA. Efficacy of autologous platelet-rich plasma combined with fractional ablative carbon dioxide resurfacing laser in treatment of facial atrophic acne scars: a split-face randomized clinical trial. Indian J Dermatol Venereol Leprol 2016;82(02):162-168

27 Leheta T, El Garem Y, Hegazy R, Abdel Hay RM, Abdel Halim D. Non-ablative 1540 fractional laser: how far could it help injection lipolysis and dermal fillers in lower-face rejuvenation? A randomized controlled trial. J Cosmet Laser Ther 2013;15(01):13-20

28 Hamra ST. Commentary on: total composite flap facelift and the deep-plane transition zone: a critical consideration in SMASrelease midface lifting. Aesthet Surg J 2016;36(05):546-549

29 Jacono AA, Bryant LM, Ahmedli NN. A novel extended deep plane facelift technique for jawline rejuvenation and volumization. Aesthet Surg J 2018;(Nov):12

30 Wulu JA, Spiegel JH. Is deep plane rhytidectomy superior to superficial musculoaponeurotic system plication facelift? Laryngoscope 2018;128(08):1741-1742

31 Maloney BP, Truswell W IV, Waldman SR. Lip augmentation: discussion and debate. Facial Plast Surg Clin North Am 2012;20 (03):327-346

32 Spiegel JH. The modified bullhorn approach for the lip-lift. JAMA Facial Plast Surg 2018;(Aug):23 